\title{
INNOVACIÓN Y CONTINUIDAD EN LOS NOMBRES PROPIOS GRIEGOS PROCEDENTES DE HISPANIA: EL CASO DE MELEPONUS Y MELPHONE *
}

\author{
ARACELI STRIANO
}

UAM

Meleponus y Melphone son dos nombres propios atestiguados en incripciones latinas procedentes de Hispania. Ambos proceden, en nuestra opinión, del adjetivo $\mu \varepsilon \lambda i ́ \varphi \omega \nu$ s, -ov 'de voz suave', con el mismo significado que $\mu \varepsilon \lambda i ́ \varphi \theta$ o $\gamma \gamma$ os, -ov, más frecuente que el anterior. Las grafías $\langle\mathrm{E}\rangle$ en lugar de $<\mathrm{I}>\mathrm{y}\langle\mathrm{P}>$ en lugar de $<\mathrm{PH}>$ en el caso de $\mathrm{Me}$ leponus pueden explicarse en términos fonéticos. En consecuencia, estos nombres, a pesar de ser

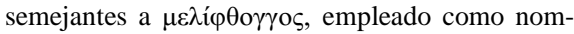
bre propio en Italia, podrían reflejar una cierta innovación y originalidad teniendo en cuenta que ninguno de los dos está atestiguado fuera de Hispania.

Palabras clave: onomástica, antroponimia griega, inscripciones latinas de Hispania.
Meleponus and Melphone are two personal names attested in Latin inscriptions coming from Hispania. Both of them derive, in our opinion, from the adjective $\mu \varepsilon \lambda i \varphi^{\prime} \omega v o \varsigma$, ,ov 'honey-voiced' with the same meaning as $\mu \varepsilon \lambda i ́ \varphi \theta o \gamma \gamma o \varsigma,-o v$, but much more infrequent. The spellings $\langle\mathrm{E}\rangle$ instead of $\langle\mathrm{I}\rangle$ and $\langle\mathrm{P}\rangle$ instead of $\langle\mathrm{PH}\rangle$ in Meleponus can be easily explained. Thus, these names, even though similar to $\mu \varepsilon \lambda i \operatorname{\varphi } \theta$ o $\gamma \gamma \circ \varsigma$, used as personal name in Italy, could reflect some kind of innovation and singularity owing to the fact that they are unattested elsewhere.

Key words: onomastics, Greek proper names, Latin inscriptions from Hispania.

1. Sabemos que una gran parte de las palabras que aparecen en las inscripciones latinas de Hispania está formada por nombres propios y dentro de éstos cabe destacar la presencia de nombres de origen griego ${ }^{1}$. La mayor

* Este trabajo se inserta en el proyecto de investigación BFF2003-04764.

1 Puede consultarse desde hace un tiempo la recopilación de estos nombres llevada a cabo por A. Lozano Velilla, Die griechischen Personennamen auf der iberischen Halbinsel, Heidelberg, 1998. Cabe señalar que algunas de las lecturas incluidas en este estudio han sido modificadas a raíz de la reedición del volumen del $C I L \mathrm{II}^{2}$ dedicado a Hispania. Hasta la fecha han salido a la luz los volúmenes del Conventus Tarraconensis (pars meridionalis) CIL $\mathrm{II}^{2}$ / 
parte de estos nombres coincide, como es natural, con los que proceden de otros lugares como Roma o la propia Grecia. Es el caso de Heliodorus, Elpis, Eugenia, Euphron, Evaristus, Demosthenes, Hermogenes, etc., que se corresponden con 'H $\mathrm{\lambda} \imath$

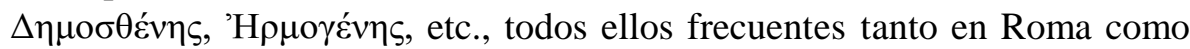
en $\mathrm{Grecia}^{2}$. Sin embargo, junto a los nombres propios (NP) que reconocemos inmediatamente, se encuentra un grupo de formas cuya interpretación no resulta tan evidente. Es el caso, por poner un ejemplo, de los antropónimos Odephorus ( CIL $\mathrm{II}^{2} / 14,400$, Sagunto, s. I) y de Laurotiche (CIL II ${ }^{2}$ 15, 936, Lora de Estepa, Sevilla, s.II) bajo cuya apariencia hemos querido reconocer la presencia de dos apelativos desconocidos hasta este momento como $\mathrm{NP}^{3}$.

2. Dentro de este último grupo de NP, pretendemos analizar dos formas concretas. Se trata del NP femenino Melphone ( CIL II 4431, Tarragona, s.II-III) y del NP masculino Meleponus ( CIL II 4118, Tarragona, aet. inc.). El primero de los nombres ha sido interpretado correctamente por A. Lozano, en nuestra opinión, quien reconoce en él una forma sincopada de Melip$h_{o n e}{ }^{4}$. Por nuestra parte, proponemos ver en el caso de Meleponus una for-

14 editado por G. Alföldy, M. Clauss, M. Olivé et al., Berlín 1995, del Conuentus Cordubensis CIL II $^{2} / 7$ editado por A. U. Stylow et al., Berlín 1995 y del Conventus Astigitanus CIL II ${ }^{2}$ 15 editado por A.U. Stylow, A. Páez et al., Berlín 1998. Resulta también muy útil poder ver el texto y las inscripciones en la página web del Centro CIL II $^{2}$ de la Universidad de Alcalá de Henares (http://www2.uah.es/imagines_cilii/) que, a su vez, se nutre de la página web del Centro de datos epigráficos de la Academia de las Ciencias de Heidelberg (Epigraphischen Datenbank Heidelberg, EDH) bajo la dirección del prof. G. Alföldy, (http://www.uni-heidelberg.de/institute/sonst/adw/edh/).

2 Cf. H. Solin, Die griechischen Personennamen in Rom. Ein Namenbuch, I, II, III.Berlín-Nueva York 1982, así como, del mismo autor, Die Stadtrömischen Sklavennamen. Ein Namenbuch, II. Griechischen Namen. Stuttgart 1996. Para la consulta de los nombres propios griegos sigue siendo de obligada referencia el manual de F. Bechtel, Die Historischen Personennamen des Griechischen bis zur Kaiserzeit. Halle 1917, además de la serie dirigida por el prof. P.M. Fraser, Lexicon of Greek Personal Names (LGPN vols. I, II, III.A y III.B) publicada en Oxford sobre cuya información, estado y volúmenes previstos puede consultarse su página web http://www.lgpn.ox.ac.uk/.

3 Cf. A. Striano «À propos de la nouvelle édition des inscriptions d'Hispania: deux notes anthroponymiques, Odephorus (Sagunto, Valence) et Laurotiche (Lora de Estepa, Sévi1le)» (en prensa).

4 «Melphone ist eine Abkürzung von Meliphone», ob. cit., p. 130. La explicación es, sin lugar a dudas, preferible a la de J. M. Abascal, Los nombres personales en las inscripciones latinas de Hispania. Murcia, 1994, p. 427, para quien Melphone respondería al femenino 
ma similar, a pesar de la grafía, es decir, equivalente a Meliphonus $^{5}$. Nos encontraríamos en ambos casos con el adjetivo $\mu \varepsilon \lambda i ́$ í $\omega v o s$, -ov 'que tiene

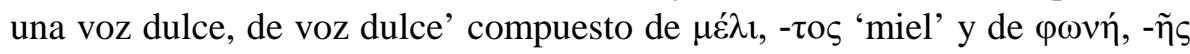
'voz', de uso poco frecuente y poético, como señalan los diccionarios al uso que mencionan únicamente un poema de Safo para ejemplificar la $\mathrm{voz}^{6}$. Junto a este adjetivo, existe otro, de significado similar y de carácter poético también (atestiguado por primera vez en una oda de Píndaro), pero, al parecer, un tanto más frecuente: se trata de $\mu \varepsilon \lambda i ́ \varphi \theta o \gamma \gamma o \varsigma$, ,ov, compuesto en su segundo elemento de $\varphi \theta$ ó $\gamma \gamma \circ \varsigma$ 'sonido articulado, voz'. Contamos con algunos NP derivados de este último adjetivo, no en territorio griego, pero sí en

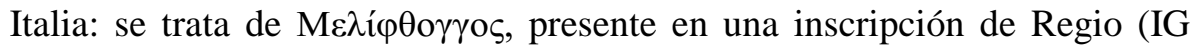
XIV 617, 8, aet. imp. ${ }^{7}$ ). Asimismo, también en Roma contamos con dos formas de este NP, Melipthongus (cf. Solin 1982, p. 713). Además, las formas simples Pthonge (Solin 1982, p. 1130) y Pthongus (cf. Solin ibid.) y lo que es para nosotros más importante, Phone (Solin 1980, p. 1130) también están presentes en Roma.

Sin embargo, carecemos de NP que ilustren las formas de Hispania Melep(h)onus, Melephone. Podríamos hallarnos, en nuestra opinión, ante dos formas ligeramente innovadoras con respecto a Roma en las que únicamente se ha sustituido el segundo término del compuesto - $\varphi \theta$ oryos por otro similar. Nos encontraríamos, en consecuencia, ante dos hapax, hecho que, sin duda, puede resultar llamativo, pero no inverosímil, dada la riqueza de los apelativos que se utilizan en la antroponimia griega ${ }^{8}$.

3. Desde el punto de vista de la grafía que presentan las formas que nos ocupan, cabría hacer algunas reflexiones. En el caso del NP femenino Melphone podríamos encontrarnos ante un hecho ocasional o esporádico de apócope de la vocal breve /i/ en interior de palabra, o bien, ante un simple error

de Melpon atestiguado en Roma (cf. Solin 1982, p. 653). Sin embargo, el NP Melpon es una forma de participio (Mé $\lambda \pi \omega v$ del v. $\mu \varepsilon ́ \lambda \pi \omega$ 'celebrar con cantos y danzas') que en femenino es

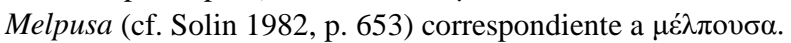

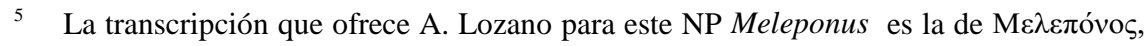
carente de paralelos en griego. Por su parte, J.M. Abascal, ob. cit. p. 427, se pregunta si el NP Meleponus pudiera estar emparentado con Melpomenus.

6 Así, en el diccionario de Bailly y en la última versión del Liddell-Scott-Jones, en el suplemento.

7 El ejemplo figura en el tomo III.A de la serie $L G P N$.

8 Basta con echar una ojeada a la sección dedicada a los "übrigen Namen" del manual de Bechtel, ob. cit., y de Solin 1982, ob. cit. 
gráfico del lapicida. En cambio, en el caso de Meleponus tenemos que dar cuenta de dos hechos: por una parte, la presencia de Mele- en lugar del esperable Meli- , y por otra, de - ponus en lugar de -phonus como en Melphone.

El hecho de que la vocal breve latina /i/ se haya abierto en /e/ justifica plenamente la fluctuación gráfica $\langle\mathrm{E}\rangle \mid\langle\mathrm{I}\rangle$ que hallamos en otros ejemplos del mismo corpus $^{9}$ y que a partir de ese momento se convierte en un hecho banal.

No ocurre lo mismo en el caso de la presencia de la grafía $\langle\mathrm{P}\rangle$ para la transcripción en la lengua latina de lo que fue en origen una consonante oclusiva labial aspirada griega $/ \mathrm{p}^{\mathrm{h}} /$. En efecto, sabemos que el sonido dejó de ser oclusivo en griego ya en época helenística y pasó a ser una consonante fricativa. Otro tanto sucedió con las otras dos consonantes aspiradas del griego, la dental y la velar. La ausencia de consonantes similares en latín provocó que en un primer momento las aspiradas griegas se transcribieran con el signo de la consonante sorda correspondiente, es decir, mediante una grafía aproximada como era la de la consonante más próxima desde el punto de vista fonético de las aspiradas. Sin embargo, con estas grafías confluyen posteriormente otras de carácter culto que pretenden reflejar (únicamente en la grafía) la aspiración de estos sonidos. Así, nos encontramos con $\langle\mathrm{PH}\rangle$, $\langle\mathrm{TH}\rangle \mathrm{y}\langle\mathrm{KH}\rangle{ }^{10}$ también en las inscripciones de Hispania, y en este contexto se justifica nuestro ejemplo femenino Melphone que acompaña a otros muchos como Stephanus, Staphylus, Theophilus, Thalamus, Chrysanthus, Chresimus, etc. ${ }^{11}$

Con todo, la consonante aspirada labial presenta en su adaptación al latín su propia particularidad debido al hecho de que la lengua latina poseía un sonido muy parecido al de la fricativa bilabial del griego como era la labiodental fricativa /f/ con la que, sin duda finalmente, se fundió e identificó plenamente. Por ello, pueden aparecer hasta tres grafías diferentes en las

9 El NP femenino atestiguado en una inscripción de Sevilla Memmesis ( Elia Memmesis, CIL II ${ }^{2}$ / 5, 966, s.III) es una buena prueba del mismo fenómeno: se trata en realidad de una grafía bajo la que se esconde Mimesis, como se interpreta en en la reedición de la inscripción. Se trata de un NP raro y poco frecuente, pero que está presente en Roma, cf. Fabiae Mimesi, Solin 1982, ob. cit., p. 1245. La transcripción de A. Lozano del NP como Mé $\mu v \eta \sigma ı \varsigma$ carece de paralelos en griego.

10 F. Biville, Les emprunts du latin au grec. Approche phonétique. Lovaina-París, 1990, pp. 139 ss.

11 Cf. A. Lozano, ob. cit., en el índice final de nombres propios. 
inscripciones latinas para la representación de este sonido: la que sería la grafía no fonética, conservadora ${ }^{12}$, paralela a la empleada para la transcripción de las otras dos aspiradas griegas que carecían de paralelos en latín $\langle\mathrm{P}\rangle$; la grafía de carácter culto paralela a $\langle\mathrm{TH}\rangle$ y $\langle\mathrm{KH}\rangle,\langle\mathrm{PH}\rangle$ y finalmente, la grafía fonética $\langle\mathrm{F}\rangle$. Los ejemplos de la alternancia de las tres grafías no son desconocidos en las inscripciones de Roma y de Hispania ${ }^{13}$.

4. Si nuestra explicación para estas dos formas fuera correcta, nos encontraríamos con hapax innovadores desde un punto de vista formal, pero

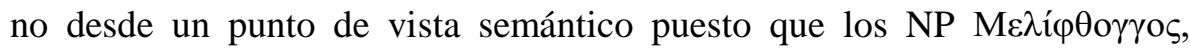

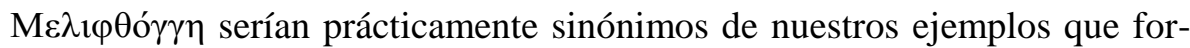
marían parte, en consecuencia, de la originalidad que puede presentar la antroponimia griega de Hispania.

12 ¿De una época en la que en griego la consonante era todavía oclusiva, o simplemente por paralelismo gráfico con las otras aspiradas?

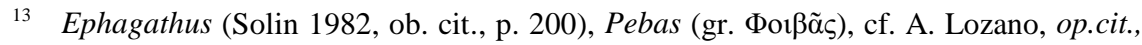
s.v. Phoebas.Este último ejemplo presentaría una grafía similar a nuestro ejemplo Meleponus: en ambos, $\langle\mathrm{P}\rangle$ serviría para la transcripción de $/ \mathrm{p}^{\mathrm{h}} \%$ 